\title{
解析航空三伤測量におけ方誤差曲面老適合する 計算の䉍易化について
}

田 浦 秀 春*

The fitting of error surface in Analytical aerial triangulation.

\section{By Hideharu Taura}

In Aerial Triangulation, the method of least squares is applied to adjust the curve of error. However this method requires much computations for complicated curves. Involving dependent and independent variables with random errors, it is believed that this theory is not logical for practical use.

This article is a study for a reasonable arithmetic process which can be used in theory and practice.

The results of our experiment shows that this new process required less computation as the use of statistics was introduced.

まえがき 航空三角測量における誤差曲面の適合に は一般に最小自乗法が用いられている。しかし最小自 乘法は複雑な曲面回帰の場合には計算量が非常に増加 するので取扱いが不便になる。また従属変数独立変数 其にランダム誤差を含んでいるから理論的にも成立し ない。一般にこのような場合には適当な数の群に別け て計算するのであるが航空三角測量では標本の数（既 知点の数）が非常に少ないからこの方法もまた適用出 米ない。そこで理論実用両面においてさらに合理的な 演算方式が開発されなければならない。小論において は比較的誤差が小さくて幾分規則性がある様に思われ る解析法の場合を主体とした。然し機械法の場合にも 現任最小自乘法の適用されている範囲では成立する筈 である。なお小論の方法を上記二方法に比べると次の 粎な特色が認められる。

1. 群別の方法に比べて計算の時間は大して変らな いが推定值の信頼区間が小さい。平均 $90 \%$ 程度 で標本の数が少ない程有利である。

2. 最小自乗法に比へて計算量ははるかに少ない。 既知点に対する適合後の残差の平方和は最小自乘 法が小さいが絶対值の和は小論の方が小さい。

3. 電子計算機のように演算の析数が制限されてい る場合には高次の最小自乘法は精度がおちるが小 諭の方法ではその心配はない。

\section{演算方式の理論}

\section{I. 直線回㷌の問題}

便宜上先ず骨子となる 2 次元の直線回帰の問題につ いて説明する。

(1) 前提条件

二つの確率度数の組 $x_{1}, \cdots, x_{n} ; y_{1}, \cdots y_{n}$ において $x_{i}$ を $N\left(X_{i}, \sigma_{x}^{2}\right) y_{i}$ を $N\left(Y_{i}, \sigma_{y}^{2}\right)$ (但し $i=1, \cdots$, $n)$ の正規分布に従うものとする。 $x_{i}-X_{i}=\varepsilon_{i}, y_{i}-$ $Y_{i}=\eta_{i}$ を以て両組の $i$ 番目の誤差と名づける。 更に次の仮定を設ける。

1. $\varepsilon_{1}, \cdots, \varepsilon_{n}$ は各々独立である。即ち

$$
E\left(\varepsilon_{i}, \varepsilon_{j}\right)=0 \quad(i \neq j)
$$

2. $\eta_{1}, \cdots, \eta_{n}$ は各々独立である。即ち

$$
E\left(\eta_{i}, \eta_{j}\right)=0 \quad(i \neq j)
$$

3. $\varepsilon_{i}$ と $\eta_{i}$ は独立である。即ち

$$
E\left(\varepsilon_{i}, \eta_{j}\right)=0 \quad(i, j=1, \cdots, n)
$$

4. $X, Y$ 間に於て次の関係が成立している。

$Y_{i}=\alpha+\beta X_{i}$
5. $\sum^{N}\left(x_{i}-x_{j}\right)$ の值は極端に0に近くならない。 $\sum$ の記号は $i>j ; i, j=1, \cdots, n$ なる組合わせの総 和を示す。

そうすると問題は $x_{1}, \cdots, x_{n} ; y_{1}, \cdots, y_{n}$ の観測值 $x_{1}^{\prime}, \cdots, x_{n}{ }^{\prime} ; y_{1}^{\prime}, \cdots, y_{n}^{\prime}$ から $\alpha, \beta, \sigma_{x}, \sigma_{y}$ を推定するこ とになる。

(2) $\alpha, \beta, \sigma_{x}, \sigma_{y}$ の推定伯 
観測值 $x_{1}{ }^{\prime}, \cdots, x_{n}{ }^{\prime} ; y_{1}^{\prime}, \cdots, y_{n}{ }^{\prime}$ を改めて $x_{1}, \cdots$, $x_{n} ; y_{1}, \cdots, y_{n}$ で表わすことにする. 任意の二組の 観測値を $\left(x_{i}, y_{i}\right),\left(x_{j}, y_{j}\right),(i>j)$ とすれば (1) の前提条件により次の関係が成立する。

$$
\begin{gathered}
\left(y_{i}-Y_{i}\right)-\beta\left(x_{i}-X_{i}\right)=\eta_{i}-\beta \varepsilon_{i} \cdots \cdots \cdots \cdots \cdot 1 \\
\left(y_{j}-Y_{j}\right)-\beta\left(x_{j}-X_{j}\right)=\eta_{j}-\beta \varepsilon_{j} \cdots \cdots \cdots \cdots 2 \\
\left(y_{i}-y_{j}\right)-\beta\left(x_{i}-x_{j}\right)-\left\{\left(Y_{i}-Y_{j}\right)-\beta\left(X_{i}-X_{j}\right)\right\} \\
\quad=\left(\eta_{i}-\eta_{j}\right)-\beta\left(\varepsilon_{i}-\varepsilon_{j}\right) \quad \cdots \cdots \cdots \cdots \cdots \cdots
\end{gathered}
$$

然るに $\left(Y_{i}-Y_{j}\right)-\beta\left(X_{i}-X_{j}\right)=0$ で且つ

$$
\left(\eta_{i}-\eta_{j}\right)-\beta\left(\varepsilon_{i}-\varepsilon_{j}\right) \text { は } N\left\{0,2\left(\sigma_{x}^{2}+\beta^{2} \sigma_{y}^{2}\right)\right\}
$$

に従うから $N$ 個の組合わせの平均をとれば $N \rightarrow$ $\infty$ に於て

$$
\begin{aligned}
& \frac{1}{2 N} \sum_{\substack{i \\
N \rightarrow \infty}}^{N}\left\{\left(y_{i}-y_{j}\right)-\beta\left(x_{i}-x_{j}\right)\right\} \\
& \longrightarrow \frac{1}{2 N} \sum_{N \rightarrow \infty}^{N}\left\{\left(Y_{i}-Y_{j}\right)-\beta\left(X_{i}-X_{j}\right)\right\}=04
\end{aligned}
$$

が成立する。故に

$$
b=\frac{\frac{1}{2 N} \Sigma\left(y_{i}-y_{j}\right)}{\frac{1}{2 N} \Sigma\left(x_{i}-x_{j}\right)}=\frac{\Sigma\left(y_{i}-y_{j}\right)}{\Sigma\left(x_{i}-x_{j}\right)} \cdots \cdots 5
$$

は $\beta$ の一致推定量になる. $\alpha$ の值は次式から直 ちに推定される。

$$
\begin{gathered}
a=\bar{y}-b \bar{x} \\
\left(\text { 但し } \bar{x}=\frac{1}{n} \sum x_{i}, \bar{y}=\frac{1}{n} \sum y_{i}, i=1, \cdots, n\right)
\end{gathered}
$$

若し $\bar{X}=\frac{1}{n} \Sigma X_{i}, \bar{Y}=\frac{1}{n} \Sigma Y_{i} \quad(i=1, \cdots, n)$ で 表わせば $\bar{x}, \bar{y}$ は夫々 $\bar{X}, \bar{Y} に$ 確率収束するから $a$ は $\bar{Y}-\beta \bar{X}=\alpha$ に確率収束する。即ち $a$ は $\alpha$ の一致推定量となる。

$\sigma_{x}{ }^{2}, \sigma_{y}{ }^{2}$ を求むるにあたっては先つ次の様な記号 を定める。

$$
\begin{aligned}
S_{x} & =\sqrt{\frac{\sum\left(x_{i}-\bar{x}\right)^{2}}{n}} \quad S_{X}=\sqrt{\frac{\sum\left(X_{i}-\bar{X}\right)^{2}}{n}} \\
S_{y} & =\sqrt{\frac{\sum\left(y_{i}-y\right)^{2}}{n}} \quad S_{Y}=\sqrt{\frac{\sum\left(X_{i}-\bar{Y}\right)^{2}}{n}} \\
S_{x y} & =\frac{\sum\left(x_{i}-\bar{x}\right)\left(y_{i}-y\right)}{n} \\
S_{X Y} & =\frac{\sum\left(X_{i}-\bar{X}\right)\left(Y_{i}-\bar{Y}\right)}{n}
\end{aligned}
$$

$S_{x}{ }^{2}, S_{y}{ }^{2}, S_{x y}$ の期待值を夫々 $E\left(S_{x}{ }^{2}\right), E\left(S_{y}{ }^{2}\right)$, $E\left(S_{x y}\right)$ で表わせば

$$
\begin{aligned}
& E\left(S_{x}{ }^{2}\right)=S_{X^{2}}+\sigma_{x}{ }^{2} \frac{n-1}{n} \quad \cdots \cdots \cdots \cdots \cdots \cdot 6 \\
& E\left(S_{y}{ }^{2}\right)=S_{Y}{ }^{2}+\sigma_{y}{ }^{2} \frac{n-1}{n} \quad \cdots \cdots \cdots \cdots \cdots \cdot 7 \\
& E\left(S_{x y}\right)=S_{X Y} \quad \cdots \cdots \cdots \cdots \cdots \cdots \cdots \cdots \cdot 8
\end{aligned}
$$

となりまた $Y_{i}=\alpha+\beta X_{i}$ なる関係式が成立して いるから

$S_{Y}=\beta S_{X} \cdots \cdots 9 \quad S_{X Y}=\beta S_{X}{ }^{2} \cdots \cdots 10$ となる， 8，9，10 から更に

$$
S_{X}{ }^{2}=\frac{E\left(S_{x y}\right)}{\beta} \cdot \cdot 11 \quad S_{Y}{ }^{2}=\beta E\left(S_{x y}\right) \cdots 12
$$

が導かれるから之を 6,7 に代入すれば

$$
\begin{aligned}
\sigma_{x}{ }^{2} & =\left[E\left(S_{x}{ }^{2}\right)-\frac{E\left(S_{x y}\right)}{\beta}\right] \frac{n}{n-1} \cdots \cdots \cdots \cdot 13 \\
\sigma_{y}{ }^{2} & =\left[E\left(S_{y}{ }^{2}\right)-\beta E\left(S_{x y}\right)\right] \frac{n}{n-1} \cdots \cdots \cdot 14
\end{aligned}
$$

となる。一方 $S_{x}{ }^{2}, S_{y}{ }^{2}, S_{x y}$ は期待値 $E\left(S_{x}{ }^{2}\right)$, $E\left(S_{y}{ }^{2}\right), E\left(S_{x y}\right)$ に確率収束しまた $b$ む $\beta$ に確 率収束するから

$$
\begin{aligned}
& {\left[S_{x^{2}}-\frac{S_{x y}}{b}\right] \frac{n}{n-1}} \\
& {\left[S_{y}{ }^{2}-b S_{x y}\right] \frac{n}{n-1} \cdots \cdots \cdots \cdots \cdots \cdots \cdots \cdots \cdots}
\end{aligned}
$$

は $\sigma_{x}^{2}, \sigma_{y}^{2}$ の一致推定量となる。

(3) $\beta$ の信頼区間

$\sigma_{y}{ }^{2}+\beta^{2} \sigma_{x}{ }^{2}$ の推定值を $S^{2}$ とすれば 15,16 式か ら

$$
S^{2}=\left[S_{x}^{2}+b^{2} S_{x}^{2}-2 b \sigma_{x y}\right] \frac{n}{n-1} \cdots \cdots \cdots \cdot 17
$$

となる.また

$$
\frac{(n-1) S^{2}}{\sigma_{y}^{2}+\beta^{2} \sigma_{x}^{2}}
$$

を考えると自由度 $(n-1)$ の $\chi^{2}$ 分布に随う。何 となれば

$$
\begin{aligned}
S_{y}^{2}= & \left.\frac{1}{n}\left\{\Sigma y_{i}-\bar{y}\right)^{2}\right\}=\frac{1}{n}\left\{\Sigma\left(\eta_{i}-\bar{\eta}\right)^{2}\right. \\
& \left.+\Sigma\left(Y_{i}-\bar{Y}\right)^{2}+2 \Sigma\left(\eta_{i}-\bar{\eta}\right)\left(Y_{i}-\bar{Y}\right)\right\} \\
S_{x}{ }^{2}= & \frac{1}{n}\left\{\Sigma\left(x_{i}-\bar{x}\right)^{2}\right\}=\frac{1}{n}\left\{\Sigma\left(\varepsilon_{i}-\bar{\varepsilon}\right)^{2}\right. \\
<\quad & \left.+\Sigma\left(X_{i}-\bar{X}\right)^{2}+2 \Sigma\left(\varepsilon_{i}-\bar{\varepsilon}\right)\left(X_{i}-\bar{X}\right)\right\} \\
S_{x y}= & \frac{1}{n}\left\{\Sigma\left(x_{i}-\bar{x}\right)\left(y_{i}-\bar{y}\right)\right\} \\
= & \frac{1}{n}\left\{\Sigma\left(\varepsilon_{i}-\bar{\varepsilon}\right)\left(\eta_{i}-\bar{\eta}\right)\right. \\
& +\Sigma\left(X_{i}-\bar{X}\right)\left(Y_{i}-\bar{Y}\right)+\Sigma\left(\varepsilon_{i}-\bar{\varepsilon}\right)\left(Y_{i}-\bar{Y}\right) \\
& \left.+\Sigma\left(\eta_{i}-\bar{\eta}\right)\left(X_{i}-\bar{X}\right)\right\}
\end{aligned}
$$

(但し $\bar{\varepsilon}=\frac{1}{n}\left(\varepsilon_{1}+\cdots+\varepsilon_{n}\right), \bar{\eta}=\frac{1}{n}\left(\eta_{1}+\cdots+\eta_{n}\right)$, $i=1, \cdots, n$ とする)

で且つ $\varepsilon_{i}$ と $\eta_{i}$ は独立であるから

$$
\begin{aligned}
\frac{(n-1) S^{2}}{\sigma_{y}^{2}+\beta^{2} \sigma_{x}^{2}} & =\frac{\sum\left\{\left(\eta_{i}-\bar{\eta}\right)-b\left(\varepsilon_{i}-\bar{\varepsilon}\right)\right\}^{2}}{\sigma_{y}^{2}+\beta^{2} \sigma_{x}^{2}} \\
& =\frac{\sum\left\{\left(\eta_{i}-b \varepsilon_{i}\right)-(\bar{\eta}-b \bar{\varepsilon})\right\}^{2}}{\sigma_{y}^{2}+\beta^{2} \sigma_{x}{ }^{2}}
\end{aligned}
$$


となるからである。次に

$$
\frac{\sqrt{3 n(n-1)}\left\{\frac{1}{2 N} \Sigma\left(x_{i}-x_{j}\right)\right\}(b-\beta)}{\sqrt{n+1} \sqrt{\sigma_{y}^{2}+\beta^{2} \sigma_{x}^{2}}} \cdot 19
$$

については

$$
=\frac{\sqrt{\frac{3(n-1)^{2}}{n+1}}\left\{\frac{1}{2 N} \Sigma\left(x_{i}-x_{j}\right)\right\}(b-\beta)}{\sqrt{S_{y}^{2}+b^{2} S_{x}^{2}-2 b S_{x y}}} \cdot 20
$$

は自由度 $(n-1)$ の $t$ 分布に随亏. 或有意水準に 相当する $t$ の值を $t_{0}$ とすれば $\beta$ の信頼区間は次 の様になる。

$$
\left.\begin{array}{c}
{\left[b-\sqrt{\frac{n+1}{3(n-1)^{2}}\left(S_{y}^{2}+b^{2} S_{x}^{2}-2 b S_{x y}\right)} \frac{t_{0}}{\frac{1}{2 N} \Sigma\left(x_{i}-x_{j}\right)},\right.} \\
\left.b+\sqrt{\frac{n+1}{3(n-1)^{2}}\left(S_{y}^{2}+b^{2} S_{x}^{2}-2 b S_{x y}\right)} \frac{t_{0}}{\frac{1}{2 N} \Sigma\left(x_{i}-x_{j}\right)}\right]
\end{array}\right\}
$$

$$
\begin{aligned}
\{ & \left.\frac{1}{2 N} \Sigma\left(x_{i}-x_{j}\right)\right\}(b-\beta) \\
= & \frac{1}{2 N}\left\{\Sigma\left(\eta_{i}-\eta_{j}\right)+\Sigma\left(Y_{i}-Y_{j}\right)\right. \\
& \left.-\Sigma\left(x_{i}-x_{j}\right) \frac{\Sigma\left(Y_{i}-Y_{j}\right)}{\Sigma\left(X_{i}-X_{j}\right)}\right\} \\
= & \frac{1}{2 N}\left[\Sigma\left(\eta_{i}-\eta_{j}\right)+\Sigma\left(Y_{i}-Y_{j}\right)\right. \\
& \left.-\left\{\Sigma\left(X_{i}-X_{j}\right)+\Sigma\left(\varepsilon_{i}-\varepsilon_{j}\right)\right\} \frac{\Sigma\left(Y_{i}-Y_{j}\right)}{\Sigma\left(X_{i}-X_{j}\right)}\right] \\
= & \frac{1}{2 N}\left\{\Sigma\left(\eta_{i}-\eta_{j}\right)-\beta \Sigma\left(\varepsilon_{i}-\varepsilon_{j}\right)\right\}
\end{aligned}
$$

之に対し群別の方法の信頼区間は

が $N\left[0, \frac{n+1}{3 n(n-1)}\left(\sigma_{y}{ }^{2}+\beta^{2} \sigma_{x}{ }^{2}\right)\right.$ の分布（証明は 付録 1) に従うから $N(0,1)$ となる。明らかに 18 と 19 は独立であるから

$$
t=\sqrt{n-1} \frac{\sqrt{\frac{3 n(n-1)}{n+1}}\left\{\frac{1}{2 N} \Sigma\left(x_{i}-x_{j}\right)\right\}(b-\beta)}{\sqrt{n-1} S}
$$

$$
\begin{aligned}
& {\left[b-\sqrt{\frac{1}{n-1}\left(S_{y}^{2}+b^{2} S_{x}^{2}-2 b S_{x y}\right)} \frac{t_{0}}{a_{1}},\right.} \\
& \left.b-\sqrt{\frac{1}{n-1}\left(S_{y}^{2}+b^{2} S_{x}^{2}-2 b S_{x y}\right)} \frac{t_{0}}{a_{1}}\right] \int^{\cdots 22} \\
& \text { (但し } a_{1}=\frac{1}{n}\left\{\left(x_{n}+\cdots+x_{m+1}\right)\right. \\
& \left.\left.-\left(x_{m}+\cdots+x_{1}\right)\right\}, m=\frac{n}{2}\right)
\end{aligned}
$$

であるから 21,22 両式を比べると

$$
\begin{aligned}
\frac{3 n}{2(n+1)} \cdot \frac{1}{2 N} \sum^{N}\left(x_{i}-x_{j}\right) \div a_{1}(\text { 証明は付䩮 } 2) & \\
\sqrt{\frac{n+1}{3(n-1)^{2}}} \cdot \frac{3 n}{2(n+1)} & =\sqrt{\frac{3 n^{2}}{4(n+1)(n-1)^{2}}} \\
& <\sqrt{\frac{1}{n-1}}
\end{aligned}
$$

随って群別の方法に比へて小論の信頼区間はその 約 $90 \%$ にたる。尚標本の数 $n$ についてこの両者 の比を作れば第一表の様になる。

第 1 表

\begin{tabular}{c|cccccccccccc}
\hline$n$ & 4 & 5 & 6 & 7 & 8 & 9 & 10 & 15 & 20 & 30 & 40 & 50 \\
\hline 比 & 0.89 & 0.88 & 0.88 & 0.88 & 0.87 & 0.87 & 0.87 & 0.87 & 0.87 & 0.87 & 0.87 & 0.87 \\
\hline
\end{tabular}

\section{（付録 1）}

$\frac{1}{2 N}\left\{\Sigma\left(\eta_{i}-\eta_{j}\right)-\beta \Sigma\left(\varepsilon_{i}-\varepsilon_{j}\right)\right\}$ の分散か： $\frac{n+1}{3 n(n-1)}\left(\sigma_{y}{ }^{2}+\beta^{2} \sigma_{x}{ }^{2}\right)$ になる証明

$\frac{N}{2}\left(\varepsilon_{i}-\varepsilon_{j}\right)=\varepsilon_{n}(n-1)+\varepsilon_{n-1}(n-3)+\cdots+\varepsilon_{\frac{2}{2}+1}-\varepsilon_{\frac{n}{2}}-\cdots-\varepsilon_{2}(n-3)-\varepsilon_{1}(n-1)$ につて考えると分散は

$2\left\{(n-1)^{2}+(n-3)^{2}+\cdots+1^{2}\right\} \sigma_{x}^{2}$ である. 然るに

$$
\begin{aligned}
& (n-1)^{2}+(n-3)^{2}+\cdots+1^{2}=\left\{(n-1)^{2}+(n-2)^{2}+\cdots+1^{2}\right\}-4\left\{\left(\frac{n-2}{2}\right)^{2}+\cdots+3^{2}+2^{2}+1^{2}\right\} \\
& \quad=\frac{1}{6} n(n-1)(n+1)
\end{aligned}
$$

であるから $\frac{1}{3} n(n-1)(n+1) \sigma_{n}^{2}$ となる。 
また $2 N=n(n-1)$ であるから $\frac{1}{2 N}\left\{\Sigma\left(\eta_{i}-\eta_{j}\right)-\dot{\beta} \Sigma\left(\varepsilon_{i}-\dot{3}_{j}\right)\right\}$ の分散は

$$
\left(\frac{1}{2 N}\right)^{2} \cdot \frac{1}{3} n(n-1)(n+1) \cdot\left\{\sigma_{y}^{2}+\beta^{2} \sigma_{x}^{2}\right\}=\frac{n+1}{3 n(n-1)}\left\{\sigma_{y}^{2}+\beta^{2} \sigma_{x}^{2}\right\} \text { となる。 }
$$

（付録 2)

$$
\begin{aligned}
& \frac{3 n}{2(n+1)} \cdot \frac{1}{2 N} \Sigma\left(x_{i}-x_{j}\right) \doteqdot a_{1} \text { の証明 } \\
& -\frac{1}{2 N} \Sigma\left(x_{i}-x_{j}\right)=\frac{1}{n(n-1)}\left\{x_{n}(n-1)+x_{n-1}(n-3)+\cdots+x_{\frac{n}{2}+1}-x_{n}-\cdots-x_{2}(n-3)-x_{1}(n-1)\right\}
\end{aligned}
$$

若し $x_{i}$ を等間隔と仮定すれば

$$
\begin{aligned}
& =\left[\left\{n(n-1)+(n-1)(n-3)+\cdots+\left(\frac{n}{2}+1\right)\right\}-\left\{\frac{n}{2}+\cdots+2(n-3)+(n-1)\right\}\right] \frac{1}{n(n-1)} \\
& =\left\{(n-1)^{2}+(n-3)^{2}+(n-5)^{2}+\cdots+1^{2}\right\} \frac{1}{n(n-1)}=\frac{1}{6}(n+1) \\
& a_{1}=\frac{1}{n}\left\{\left(x_{n}+x_{n-1}+\cdots+x_{m+1}\right)-\left(x_{m}+\cdots+x_{2}+x_{1}\right)\right\} \\
& x_{i} \text { を等間隔と仮定すれば } \\
& =\frac{1}{n}\left\{n+(n-1)+\cdots+\left(\frac{n}{2}+1\right)\right\}-\left\{\frac{n}{2}+\cdots+2+1\right\}=\frac{n}{4}
\end{aligned}
$$

故に $\frac{(\mathrm{B})}{(\mathrm{A})}=\frac{3 n}{2(n+1)}$ となる。

（4） $\beta$ が既知の場合における $\alpha$ の信頼区間

比の場合に於て $\alpha$ の推定值は次の式で与えられる。

$$
a=\bar{y}-\beta \bar{x}
$$

処か： $a-\alpha=(\bar{y}-\bar{Y})-\beta(\bar{x}-\bar{X})=\bar{\eta}-\beta \bar{\varepsilon} \quad$ であるから

$$
\frac{\sqrt{n}(a-\alpha)}{\sqrt{\sigma_{y}^{2}+\beta^{2} \sigma_{x}^{2}}}
$$

は $N(0,1)$ に随う。且つ 18 と 24 は独立であるから $t=\sqrt{n-1} \frac{\sqrt{n}(a-\alpha)}{\sqrt{n-1} S}=\frac{\sqrt{n-1}(a-\alpha)}{\sqrt{S_{y}{ }^{2}+\beta^{2} S_{n}{ }^{2}-2 \beta S_{x y}}}$ は 自由度 $(n-1)$ の $t$ 分布に随了。或有意水华に相当する $t$ の值を $t_{0}$ とすれは $\alpha$ の信頼区間は $\left[a-\frac{1}{\sqrt{ } n-1} \sqrt{S_{y}{ }^{2}+\beta^{2} S_{x}{ }^{2}-2 \beta S_{x y}} t_{0}, \quad a+\sqrt{\sqrt{n-1}} \sqrt{S_{y}{ }^{2}+\beta^{2} S_{x}{ }^{2}-2 \beta S_{x y} t_{0}}\right]$ で表わされる。

(5) 演 算

$n$ 租の穓測值を $\left(x_{1}, y_{1}\right), \cdots,\left(x_{n}, y_{n}\right)$ とする。 $\sum^{N}\left(y_{i}-y_{j}\right)$ を求むるには次の样に展開すれは計算が簡単にな る。

$$
\begin{aligned}
\sum^{N}\left(y_{i}-y_{j}\right)= & \left\{(n-1) y_{1}+(n-2) y_{n-1}+(n-3) y_{n-2}+\cdots+3 \cdot y_{4}+2 \cdot y_{3}+y_{2}\right\} \\
& -\left\{(n-1) y_{1}+(n-2) y_{2}+(n-3) y_{3}+\cdots+3 y_{n-3}+2 y_{n-2}+y_{n-1}\right\}
\end{aligned}
$$

同栐にして $\sum^{N}\left(x_{i}-x_{j}\right)$ を求める。

5 式から $b=\frac{\sum\left(y_{i}-y_{j}\right)}{\sum\left(x_{i}-x_{j}\right)}$ として $\beta$ の推定值が決定される。

(6) 計算例

第二表の粎な锤則做に対し直線を適合する。各種の方法を比較すると次の様になる。

第 2 表

\begin{tabular}{l|cccccccccc}
\hline 区 $_{\text {分 }}^{\text {数 }}$ & 1 & 2 & 3 & 4 & 5 & 6 & 7 & 8 & 9 & 10 \\
\hline$X$ & 2.15 & 2.54 & 2.74 & 2.98 & 3.07 & 4.18 & 5.06 & 5.08 & 6.55 & 6.84 \\
\hline$Y$ & 3.68 & 4.08 & 3.98 & 4.53 & 4.82 & 6.14 & 7.49 & 8.22 & 9.68 & 9.94 \\
\hline
\end{tabular}


本例は“化学および化学工業における統計解析”

(C. A. Bennett, N. L. Franklin 其著) の 6.3 .表の 一部をとったものである。

イ. 群別の方法

回㷌 直線 $y=0.614+1.338 x$

残差の平方和 0.56 残差の絶対值の和 1,90

口. 最小自乘法

回㷌直線 $y=0.588+1.344 x$

残差の平方和 0.56 残差の絶対偭の和 1,89

\section{八. 小論の方法}

回 㥂 直 線 $y=0.542+1.355 x$

残差の平方和 0.56 残差の絶対值の和 1,86

\section{II. 平面回帰の問題}

2 次元の直線回帰の理論をその㒒抬㲀すればよい。 即ち $Z_{i}=\alpha+\beta X_{i}+\gamma Y_{i}$ の関係が成立しているもの として $\alpha ; \beta, \gamma$ の推定値を夫々 $a, b, c$ とすれば次の 样な演算が成立する.（証明は省略する）

1. $n$ 組の锤測值を $\left(x_{1} \quad y_{1} z_{1}\right), \cdots,\left(x_{n} y_{n} z_{n}\right)$ とす る。

2. 之等の標本を $x_{i}$ について大きい制序に並・か える。

3. 2 の順序に基いて $\Sigma\left(x_{i}-x_{j}\right), \Sigma\left(y_{i}-y_{j}\right), \Sigma$ $\left(z_{i}-z_{j}\right)$ を二変数の場合の 27 式の要領て計算す る。

4. 次に之等の標本を $y_{i}$ について大きい順序に並 べかえる。

5. 4 の順序に基いて $\sum\left(x_{i}^{\prime}-x_{j}^{\prime}\right), \Sigma\left(y_{i}^{\prime}-y_{j}^{\prime}\right)$, $\Sigma\left(z_{i}^{\prime}-z_{j}^{\prime}\right)$ を計算する。

6. 次の連立方程式を解いて $\beta, \gamma$ の推定值の $b, c$ を求める。

$$
\begin{aligned}
& b \cdot \Sigma\left(x_{i}-x_{j}\right)+c \cdot \Sigma\left(y_{i}-y_{j}\right)=\Sigma\left(z_{i}-z_{j}\right) \\
& b \cdot \Sigma\left(x_{i}^{\prime}-x_{j}^{\prime}\right)-c \cdot \Sigma\left(y_{i}^{\prime}-y_{j}^{\prime}\right)=\Sigma\left(z_{i}^{\prime}-z_{j}^{\prime}\right)
\end{aligned}
$$

7. $\alpha$ の推定储 $a$ は次の式から計算する。 $a=\bar{z}-b \bar{x}-c \bar{y}$

\section{III. 解析航空三角測量における誤差曲面の 回帰問題}

平面回㝑の問題は更に $n$ 次元線型回㷌の問題に拡張 出来る。然し航空三角測量の䛊差曲面は 2 次の項を含 んているから無条件には挔張出来ない。幸い解析航空 三角測量に於ては 2 次の項の影辢度は極めて小さいか ら 1 次の項同様正規分布に随うものと見なして差支え なさそうである。そうすると $\boldsymbol{n}$ 次元線型回㷌の問題と して取扱うことが出来る（この仮定については最小自 乘法の場合も群別の場合も全く同じである)。演算方 式は 3 次元の場合をその俧捄张すればよいから省略す る。

$\mathrm{M}$ 地区の作業に於て第三表の栏なデータを得たの でにに次の様な誤差曲面を適合した. 但し $X, Y$ は機 極坐標 $\Delta X$ は测地座標とする。

$\Delta X=A+B X+C Y+D X^{2}+E X Y$

1. 小論の方法

$$
\begin{array}{ll}
A=-12.232 & B=4.307675996 \\
C=0.443979117 & D=-0.029006526 \\
E=0.028877798 &
\end{array}
$$

口. 最小自乘法

$$
\begin{array}{ll}
A=-11.533 & B=4.111038490 \\
C=0.218516138 & D=-0.018706906 \\
E=0.050460071 . &
\end{array}
$$

結果的にみて線型回㷌の場合と同様適合後の残差の 平方和は最小自乘法が小いか絶対值の和は小論の方 が小さい。然し何れにせよ大した差異は認められな W。

計算量に於ては問題にならぬ程小論の方が小さい。

\begin{tabular}{|c|c|c|c|c|c|c|c|c|c|c|c|c|}
\hline 区 分 & 1 & 2 & 3 & 4 & 5 & 6 & 7 & 8 & 9 & 10 & $\begin{array}{l}\text { 絶対值 }^{\text {の 和 }} \\
\end{array}$ & $\mid \begin{array}{l}\text { 絶対俌の } \\
\text { 平方 和 }\end{array}$ \\
\hline 旡 & 0.13 & -0.10 & -0.61 & 0.27 & 0.64 & -0.01 & -0.03 & -0.11 & -0.04 & -0.14 & 2.08 & 0.91 \\
\hline 最小自乗法 & 0.10 & 0 & -0.36 & 0.13 & 0.48 & -0.25 & -0.22 & -0.25 & 0.27 & -0.03 & 2.12 & 0.63 \\
\hline
\end{tabular}

あとがき 小論中の二三の計算例にも分る様に面 適合後の残差は平方和に於て最小自乗法が传れている か絶詨低の和に於ては小論の方が優っている。随って [P. 47 へつつく]

第 3 表

\begin{tabular}{c|cccccccccc}
\hline 区 & \multicolumn{1}{c|}{1} & 2 & 3 & 4 & 5 & 6 & 7 & 8 & 9 & \multicolumn{1}{c}{10} \\
\hline$\Delta X$ & 0 & 3.74 & 0.27 & 6.23 & 18.52 & 29.24 & 36.07 & 40.53 & 50.55 & \multicolumn{1}{c}{49.04} \\
$X$ & 2.768 & 3.777 & 2.830 & 4.290 & 7.868 & 10.176 & 12.379 & 13.206 & 16.130 & 16.155 \\
$Y$ & 1.254 & 0.036 & -0.126 & 1.380 & -1.055 & 0.860 & -0.757 & 0.997 & 0.882 & -0.981 \\
\hline
\end{tabular}

第 4 表 